\title{
PAPER \\ Interactive Learning of Spoken Words and Their Meanings Through an Audio-Visual Interface
}

\author{
Naoto IWAHASHI ${ }^{\dagger a)}$, Member
}

\begin{abstract}
SUMMARY This paper presents a new interactive learning method for spoken word acquisition through human-machine audio-visual interfaces. During the course of learning, the machine makes a decision about whether an orally input word is a word in the lexicon the machine has learned, using both speech and visual cues. Learning is carried out on-line, incrementally, based on a combination of active and unsupervised learning principles. If the machine judges with a high degree of confidence that its decision is correct, it learns the statistical models of the word and a corresponding image category as its meaning in an unsupervised way. Otherwise, it asks the user a question in an active way. The function used to estimate the degree of confidence is also learned adaptively on-line. Experimental results show that the combination of active and unsupervised learning principles enables the machine and the user to adapt to each other, which makes the learning process more efficient.

key words: mutli-modal interface, word acquisition, multi-media processing, interactive learning, active learning, word meaning
\end{abstract}

\section{Introduction}

The progress of sensor technologies and the infrastructure of ubiquitous computing have enabled machines to sense physical environments as well as the behaviour of users. In the near future, machines that change their behaviour according to the situation will become more and more common, and they will support human activities in everyday life. Such machines include robots, wearable devices, and so on. In order to be truly useful, they should also have user-centered intelligent interfaces. One way to obtain such interfaces is through personalization. One of the basic and most essential features of personalized multimodal interfaces is the ability of the machine to share its environment and experiences with the user. In the future, spoken language interfaces will become increasingly important not only because they enable hands-free interaction but also because of the nature of language, which inherently conveys meaning based on shared experiences and knowledge [1]. In order for us to take advantage of this nature of language in the personalization of multimodal interfaces, language processing methods must make it possible to reflect both shared environments and dynamically changing shared experiences.

However, existing language processing methods, which are characterized by fixed language knowledge, do

Manuscript received January 22, 2007.

Manuscript revised October 7, 2007.

${ }^{\dagger}$ The author is with National Institute of Information and Communications Technology, Keihanna Science City, 619-0288 Japan. Also with Advanced Telecommunications Research Institute International, Keihanna Science City, 619-0288 Japan.

a) E-mail: naoto.iwahashi@ atr.jp

DOI: 10.1093/ietisy/e91-d.2.312 not satisfy this requirement. In these methods, information is represented and processed by symbols whose meaning has been pre-defined by the developers of the machines. In most cases, the meaning of each symbol is defined by its relationship with other symbols, and it is not connected to the physical world. The precise nature of experiences shared by a user and a machine, however, depends on the specific situation. Because it is impossible to prepare symbols for all possible situations in advance, machines cannot appropriately express and interpret experiences under dynamically changing situations. As a result, users and machines fail to interact in a way that appropriately reflects shared experiences.

To overcome this problem, the machines themselves must be able to create internal symbols reflecting shared experiences in natural ways. Such symbols may include spoken words, categories of different objects, or categories of user behaviour; symbols referring to the relationship among different objects; and symbols referring to different situations.

\section{Spoken word acquisition}

This paper focuses on the acquisition of spoken words and visual categories as their meanings through an audio-visual interface in order to develop machines that can communicate with users in everyday-life environments. Such machines should acquire new words with the minimum number of interactions with users. In general, the difficulty of acquiring spoken words and the visual objects they refer to as their meanings can be ascribed to the difficulty of the specification of features and the difficulty of the extension of them.

Specification: The acoustic features of a spoken word and the visual features of an object it refers to should be specified using spatiotemporally continuous audiovisual data. For speech, this means that a continuouslyspoken utterance is first segmented into intervals, and then acoustic features are extracted from one of the segmented intervals. For objects, this means that an object is first selected for a given situation, and then the spatial part of the object is segmented; after that visual features are extracted from the segmented part of the object.

Extension: Both the acoustic and the visual features specified should be extended in order to create categories for a given word and its meaning. Extension of the features of a referent of a word to form the meanings of the word has been investigated through psychological ex- 
periments [2]. When shown an object and given a word for it, human subjects tend to extend the features of the referent immediately to assume a particular meaning of the word; this cognitive ability is called fast mapping. For machines, however, the difficulty in acquiring spoken words arises not only from the difficulty in extending the features of referents but also from the difficulty in understanding spoken words, because the accuracy of speech recognition by machines is currently much lower than that by humans, which means that it is not easy for the machines to determine whether two different speech segments belong to the same word category.

\section{Interactive learning}

This paper mainly addresses the problem of extension. It proposes a new method for spoken word acquisition in which learning is carried out in an interactive way, rather than in a batch way. The advantages of interactive learning in spoken word acquisition tasks are summarized below.

(1) Additional information. If the machine does not have enough information to make an accurate decision about learning, it can ask the user for additional information.

(2) Adaptation to users. The machine can adapt its behaviour efficiently to the learning conditions given by the user, such as speech individuality, word meanings to be learned, characteristics of objects, and lighting conditions.

(3) Adaptation to machines. The user can understand the machine's state of learning from its responses and can change behaviour accordingly to make the learning more efficient.

\section{Outline}

This paper proceeds as follows. Section 2 describes related works and the differences between previous methods and the method proposed in this paper. Section 3 describes a spoken word acquisition task used in this study. Section 4 describes a statistical model of the lexicon that the machine acquired in the experiments. Section 5 describes the data set used in the experiments. Sections 6, 7, 8, and 9 describe the proposed interactive learning method and its evaluation. Section 10 discusses the advantages, limitations, and future applications of the developed method.

\section{Related Works}

There have been a number of studies on language acquisition in which the learning proceeded from input speech and its referents. Gorin and colleagues [3] developed a method which enables a machine to map the user's speech onto its call routine actions in telephone service using spoken utterances with labels for each corresponding action. In their study, utterances were spoken with pauses between words. Nakagawa and co-workers [4] developed a method of learning from images and speech input describing each image. The images in their study were those of blocks with clearly distinct geometrical shapes such as triangles, rectangles, and circles, and these images were recorded by a video camera. The speech input was a continuous stream of speech without pauses between words. This was the first method in which learning occurred from sensory input only and with continuously spoken utterances. This method, however, used classifiers prepared beforehand to extract discrete semantic attributes from each image. In contrast, Akaho and colleagues [5] described a method which enables learning the connections between speech and object images without any given classifiers. The method provides for the learning of direct mappings between speech signals and visual objects, and it does not require the construction of any categories for words or images. Roy [6] described a computational model of language acquisition by infants. In his model, the categories of both spoken words and object images as well as the connections between them were learned simultaneously from noisy audio-visual sensory input which also included irrelevant data. In this model, each image category was represented through only one non-linguistic feature channel for either colour or shape. This was the first method that made it possible to process data obtained in the observations of communication between infants and caregivers in an actual environment. Gorin and colleagues [7] extended their earlier method to handle continuously spoken utterances. This method made it possible to learn the mapping from users' naturally spoken utterances to the call routine actions the users ask for in telephone service. In the learning, salient phoneme sequences were extracted automatically without any pre-defined lexicon. The methods developed by Roy et al. and Gorin et al. used previously given phoneme classifiers, and speech input was converted into phoneme sequences based on these classifiers. In contrast, Iwahashi [8] developed a method of learning without the use of phoneme classifiers prepared in advance. The method enables a simultaneous learning of phoneme-like speech units, words, and their meanings. In the method, word meanings are represented through multiple non-linguistic feature channels for shape, colour, and size. This method uses not only static images like other methods do, but also dynamic images showing the movements of objects. Inamura and colleagues [9] and Steels and Kaplan [10] developed methods that enable interactive learning of image categories as the meanings of words in a predefined vocabulary. Dusan and Flanagan [12] described the use of word acquisition methods to enhance multimodal interfaces with speaking, pointing with a mouse, typing, drawing and capturing images with a CCD camera; the researchers used a task of graphic drawing on a computer screen to investigate whether the interfaces could be improved by embedding the methods in which users could teach a computer new words for graphical objects on the screen and operate the graphical objects. In results, it was confirmed that the users could easily teach the computer eight or nine words in about ten minutes. Ballard and $\mathrm{Yu}$ [13] used a word acquisition method for interfaces designed to enable machines to learn new words to describe user actions in everyday life, such as stapling papers. Taguchi and colleagues [11] developed a learning method 
for image concepts that uses the biases observed in the language development of children.

The method proposed in this paper is different from the above methods in that it enables the learning of new spoken words and image categories as their meanings incrementally, in an interactive way, without the use of a pre-defined vocabulary. It is based on a combination of principles for active and unsupervised learning, and it cnables the user and the machine to adapt to each other in a natural way, which makes the learning more efficient [14].

\section{Interactive Spoken Word Acquisition}

\subsection{Task Set-up}

In order to implement basic and essential principles of spoken word acquisition, a simple interactive procedure was developed in this study. The setting for the procedure is shown in Fig. 1. The procedure involves the following steps. The user puts a physical object on a table in front of a stereo camera and at the same time says the name of the object or its description into a close-talk microphone. The machine makes a decision about whether the input word is one of the words in its vocabulary (whether it is a known word) or not (whether it is an unknown word). If the machine judges that the input word is an unknown word, it enters the word into its vocabulary. If the machine judges that it cannot make an accurate decision, it asks the user a question about whether the input word is part of its vocabulary. In response, the user answers yes or no. By repeating this interaction, the machine learns spoken words and image categories as their meanings incrementally, on-line.

In our experiments described in this paper, when the machine asked questions, it output the word said by the user, which had been pre-recorded during the course of learning. This was taken to mean "Did you say this word?" The user's response was input through a keyboard. This set-up, which may seem fairly simple, is enough to investigate the validity of the proposed methods on requirements in implementing spoken language acquisition tasks. In developing a practical system, this simple set-up can be easily extended to more sophisticated tasks. For instance, the keyboard input can be replaced with a speech interface.

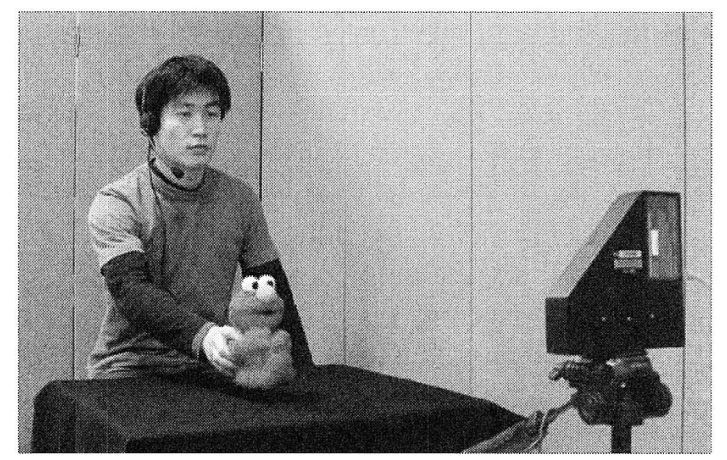

Fig. 1 The user is putting an object in front of a stereo camera while saying a word describing the object.

\subsection{Requirements and Approaches}

A word acquisition task must satisfy the following requirements.

Decisions about unknown words: Even state-of-the-art speech recognizers are not very accurate in recognizing phoneme sequences without high-order linguistic constraints. Using ambiguous output from such a speech recognizer, the machine has to make a correct decision about whether the input word is one of the words in its vocabulary.

Confidence estimation: The machine must estimate the degree of confidence that its decision about an unknown word is correct in order to control the learning interaction automatically. In addition, the mechanism for this confidence estimation must be adapted to given learning conditions including speech individuality, lighting conditions, and characteristics of the objects used.

The method presented in this paper satisfies the above requirements with the following features.

Use of speech and visual information: It uses not only speech but also visual information about the objects to make an accurate decision about an unknown word. For example, when the user shows an orange and says the word /orin $3 /$, even if the speech recognizer outputs an unknown word /areiz/ as the first candidate, the system can modify it to the correct word / $/$ rin $_{3} /$ in the lexicon.

Active and unsupervised learning: The degree of confidence for decisions about unknown words is estimated statistically. Both the function for estimating the degree of confidence and the lexicon are learned incrementally, on-line, based on a combination of active and unsupervised learning principles.

\section{A Statistical Model of the Lexicon}

\subsection{Speech and Visual Features}

Speech was detected and segmented based on changes in the short-time power of speech signals. Objects were detected when they were located at a distance of $50-80 \mathrm{~cm}$ from the stereo camera unit. All speech and visual sensory output was converted into predetermined features. The speech features used were Mel-frequency cepstral coefficients [15], which are based on short-time spectrum analysis; their delta and acceleration parameters; and the delta of short-time log power. These features were calculated in 20-ms intervals with a 30-ms-wide window. The visual features used were $\mathrm{L} * \mathrm{a} * \mathrm{~b} *$ components (three dimensions) for the colour, complex Fourier coefficients (eight dimensions) of 2D contours for the shape [16], and the area of an object (one dimension) for the size. 


\subsection{Representation of Words}

Each word, or a lexical item, to be learned included statistical models for the spoken word and an image category for its meaning. The model for each image category was represented by a Gaussian function in a twelve-dimensional visual feature space. Here, the covariance parameters of the Gaussians were fixed to 0 to prevent over-learning by a small number of learning samples, and the mean vectors and variance vectors were learned. When a word means the colour of objects, the variances of the colour components should be small, and those of the other components large. The same holds for shape and size. In contrast, when a word means an object name, the variances of all components should be small. The model for each spoken word was represented by a concatenation of hidden Markov models (HMMs) for sub-word units.

\subsection{Sub-Word HMMs}

Sub-word HMMs were learned in an unsupervised way in advance. Each sub-word HMM included three states and allowed for left-to-right transitions. Twenty sub-word HMMs were connected with one another to construct a whole subword HMM (Fig. 2) in which transitions were allowed from the last states of the sub-word HMMs to their first states. All parameters of this HMM were learned using speech data approximately one minute in length without any transcriptions. The speech data were input orally by the same user who would later teach the machine in the experiments with interactive spoken word acquisition. The learning was performed using the Baum-Welch algorithm [18]. After learning sub-word HMMs, the individual sub-word HMMs, $h_{A}$, $h_{B}, h_{C}, \ldots, h_{T}$, were separated from one another by deleting edges between them, and a sub-word HMM set, $H$, was constructed. The number of sub-word HMMs was determined empirically. Our spoken word acquisition method used these sub-word HMMs.

Nakagawa and colleagues [17] also investigated a

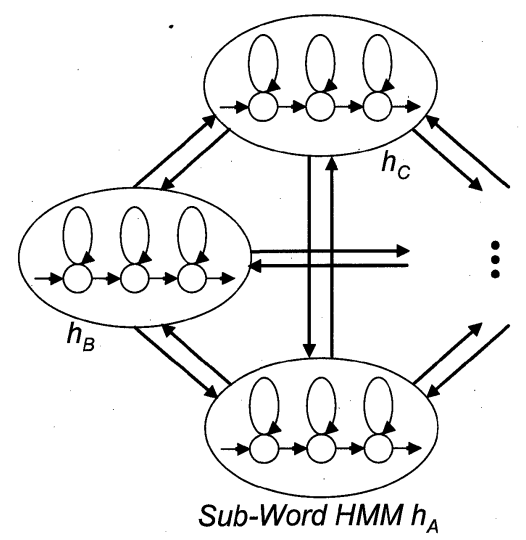

Fig. 2 Structure of the HMMs for unsupervised learning of sub-word models. method for learning speech units by HMMs in the context of improving speech recognition performance. The learning method determined the number of states of each subword HMM automatically, and it was based on the sub-word HMM sequences obtained by the Viterbi algorithm using information on the word identity of speech. In contrast, the method presented here uses a fixed number of states of the sub-word HMM, and it does not use the Viterbi algorithm or any information on the word identity of speech.

\section{Data Materials}

Although our spoken word learning method is interactive and enables learning on-line, pairs of spoken words and object images were recorded in advance as training data to improve the efficiency and consistency of the experiments.

Forty-five objects were used. They were stuffed toys and blocks. Multiple images of each object were recorded using a stereo camera. The images of the objects were taken under different lighting conditions and at a different position from the camera. As a result, the images of the same objects looked quite different in terms of colour, shape, and size.

In addition, forty words that described the objects were chosen, and they were repeated into a microphone by a male speaker seven times. Speech was recorded using a close-talk microphone in an office environment. The selected words were thirty-two nouns, which were object names, and eight adjectives, each describing an attribute of one object. The adjectives were Japanese words for red, blue, yellow, big, small, circular, triangular, and rectangular. Some object names referred to several stuffed toys which were very similar in shape and colour but very different in size.

The data set $X=\left\{\left(s_{1}, v_{1}\right),\left(s_{2}, v_{2}\right), \ldots,\left(s_{280}, v_{280}\right)\right\}$ consisted of speech-image pairs in which speech samples $s_{i+1}$, $\ldots$ and $s_{i+7}$ were samples of the same word for $i=0, \ldots 39$, that is, each set of seven consecutive speech-image samples corresponded to the same word. This data set was used in the interactive word acquisition experiments. Figure 3 shows some of the objects we used along with their names and some of their attributes.

\section{Unknown-Word Decisions Based on Speech and Vi- sual Information}

As described in Sect. 3.2, making decisions about unknown words is an important part of interactive spoken word acquisition. The difficulty arises mainly from the inaccuracy of speech recognizers. To investigate the accuracy of speech recognition in our task, an experiment was conducted in which speech samples in the learning data $X$ were recognized using a learned subword unit HMM set $H$ with no constraints on the transitions between the subwords. Seventynine different subword sequences were output by a speech recognizer for two-hundred eighty samples, which included forty words. In each of twenty-nine of the words, the outputs for the samples were not identical. Table 1 shows examples of output subword sequences for such words. Note 


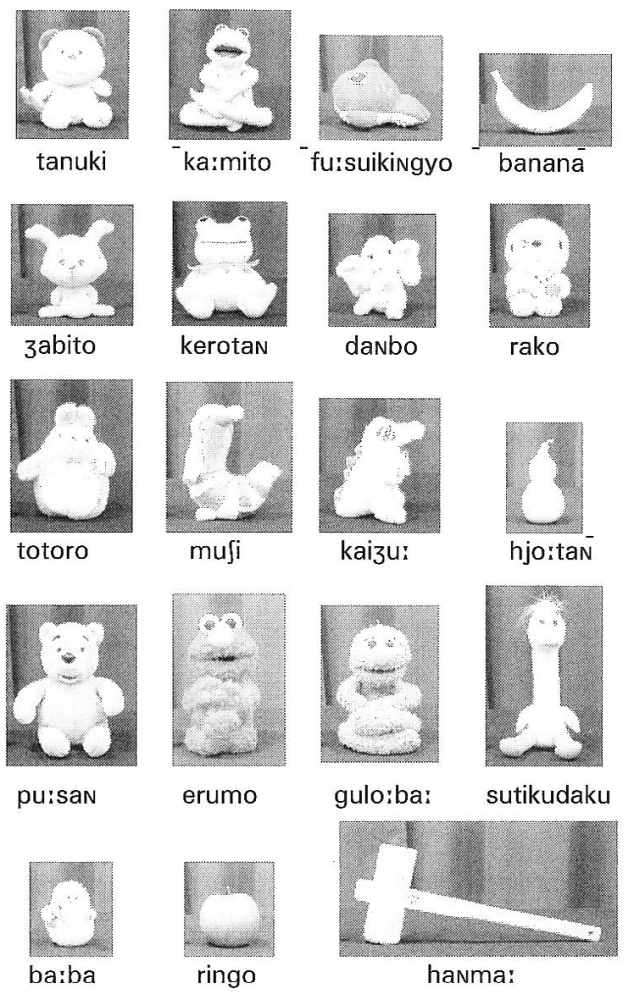

(a)
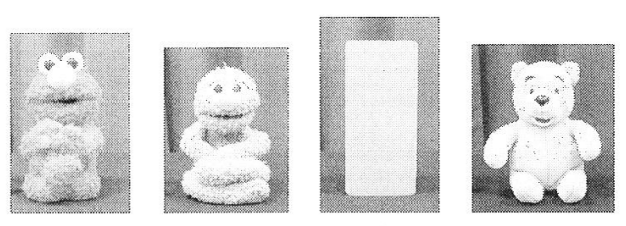

o:ki: (big)
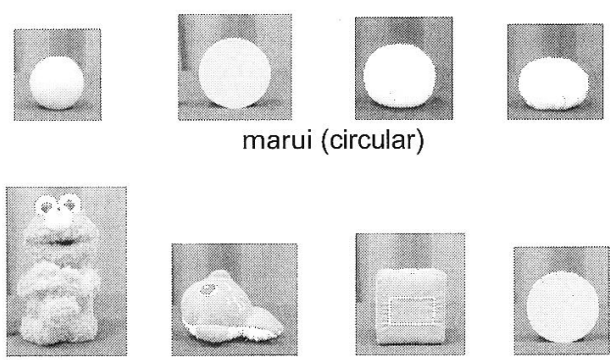

aka (red)

(b)

Fig. 3 Examples of objects used in the experiments along with (a) their names and (b) their attributes.

Table 1 Examples of output subword sequences.

\begin{tabular}{|l|l|}
\hline pronunciation & subword sequences \\
\hline \hline banana & $\begin{array}{l}\text { IRPNIDIR, NINPNIDIR, RPNIDIR, } \\
\text { RPNIDNIR }\end{array}$ \\
\hline mikan & JGTIDG, NJGTIDG, JGTIDNG \\
\hline biguba:do & $\begin{array}{l}\text { HGTCNIPNK, THGTGIPNK, } \\
\text { THGTCNIDPNK }\end{array}$ \\
\hline
\end{tabular}

that the alphabetic letters in the subword sequences in Table 1 represent the subword HMMs; they are not phonetic symbols. This experiment demonstrates that it is difficult

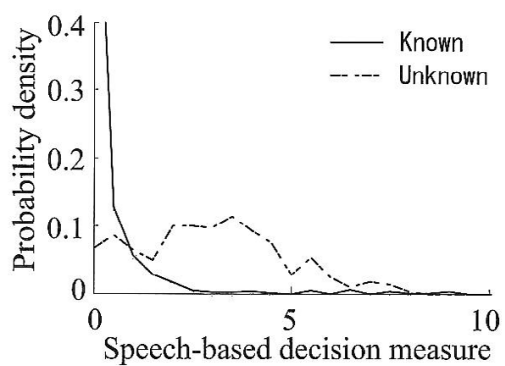

(a)

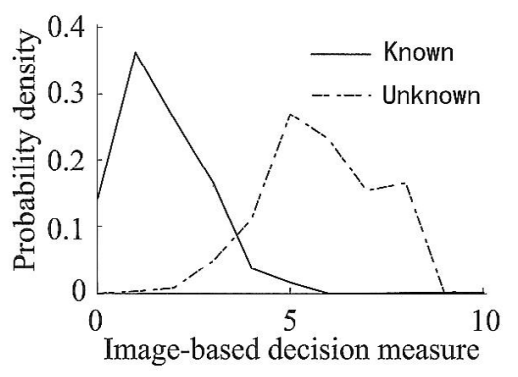

(b)

Fig. 4 Distributions of values of (a) speech-based decision measure and (b) image-based decision measure.

for a machine to make accurate decisions about unknown words bascd solcly on specch information.

In the proposed method, decisions about unknown words are made by using not only speech but also visual information. The measure $H_{s}(s)$ for such a decision given a speech feature $s$ is the $\log$ ratio of the maximum value among the likelihood values of all possible sub-word HMM sequences to the maximum value among the likelihood values of all sub-word HMM sequences for the words in the lexicon, and it is written as

$$
H_{s}(s)=\max _{q} \log p\left(s ; \Lambda_{q}\right)-\max _{i} \log p\left(s ; \Lambda_{u_{i}}\right)
$$

where $q$ and $\Lambda_{q}$ denote the sequence of sub-word HMMs and the parameters of concatenated HMMs, respectively. $u_{i}$ denotes the sequence of sub-word HMMs for the $i$ th word in the lexicon.

The measure $H_{v}(v)$ for a decision about an unknown word given a visual feature $v$ is the output $p\left(v ; g_{i}\right)$ of a Gaussian function representing the meaning of the word $w_{i}$, where $g_{i}$ denotes the parameter set of the Gaussian function, and it is written as

$$
H_{\nu}(v)=-\max _{i} \log p\left(v ; g_{i}\right) .
$$

Distributions of the values of these measures were calculated by using the data set $X$ for cases when the machine was assumed to have made a decision that the speech-image input $(s, v)$ was for a known word correctly and when it was assumed to have made a decision that the input was for an unknown word correctly, and these are shown in Fig. 4. In these calculations, spoken word and image models were used, which were learned by the machine in advance by using the data set $X$ with correct word labels given manually. 


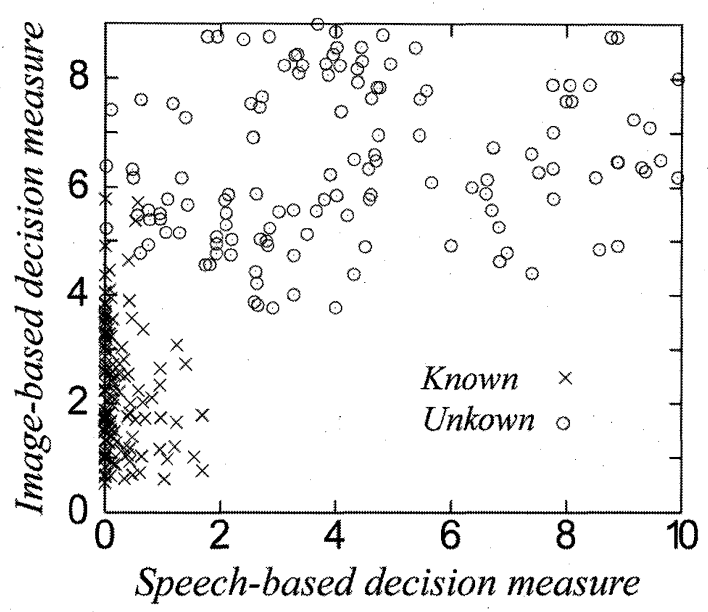

Fig. 5 A joint distribution of values of the speech-based and image-based measures.

Figure 5 shows a joint distribution of the values of the speech-based and image-based decision measures. As can be seen in the figure, there is a clear-cut slant boundary between the areas representing the known and unknown word conditions. This result suggests that a simultaneous use of both speech-based and image-based measures is most effective.

Note that the word labels used to obtain the results shown in Figs. 4 and 5 were not used in the interactive spoken word acquisition method described in the following sections.

\section{Active and Unsupervised Learning Algorithm}

Interactive word acquisition was carried out by using the algorithm shown in Fig. 6 as well as both the speech-based decision measure, $H_{s}(s)$, and the image-based decision measure, $H_{v}(v)$. Let $L=\left(m,\left(\Lambda_{1}, g_{1}\right), \ldots\left(\Lambda_{m}, g_{m}\right)\right)$ denote the machine's lexicon, where $m$ is the number of lexical items in the lexicon. Let $\Lambda_{j}$ and $g_{j}$ denote, respectively, the HMMs for the spoken word and the Gaussian function for the visual category of the $j$ th lexical item. When the $i$ th speech sample, $s_{i}$, and its referent image, $v_{i}$, were input by the user during the course of interactive learning, the machine updated its lexicon by creating a new lexical item or by updating an existing lexical item to which the input samples were assigned. Learning was done on-line and incrementally. Batch-mode learning was not used for image categories. The decision on whether to create a new lexical item or to update an existing lexical item was made in either an active or an unsupervised way. In the unsupervised mode, it made an independent decision about whether the input speech was a word in its lexicon or not. In the active mode, it asked the user a question. The choice between the modes was made based on the confidence function $F$, which output the degree of confidence that the input speech-image pair represented one of the items in the machine's lexicon. The function $F$ is written as

$$
F(s, v)=\frac{1}{1+\exp \left(-\alpha_{1}\left(H_{s}(s)-\beta_{1}\right)\right.}
$$

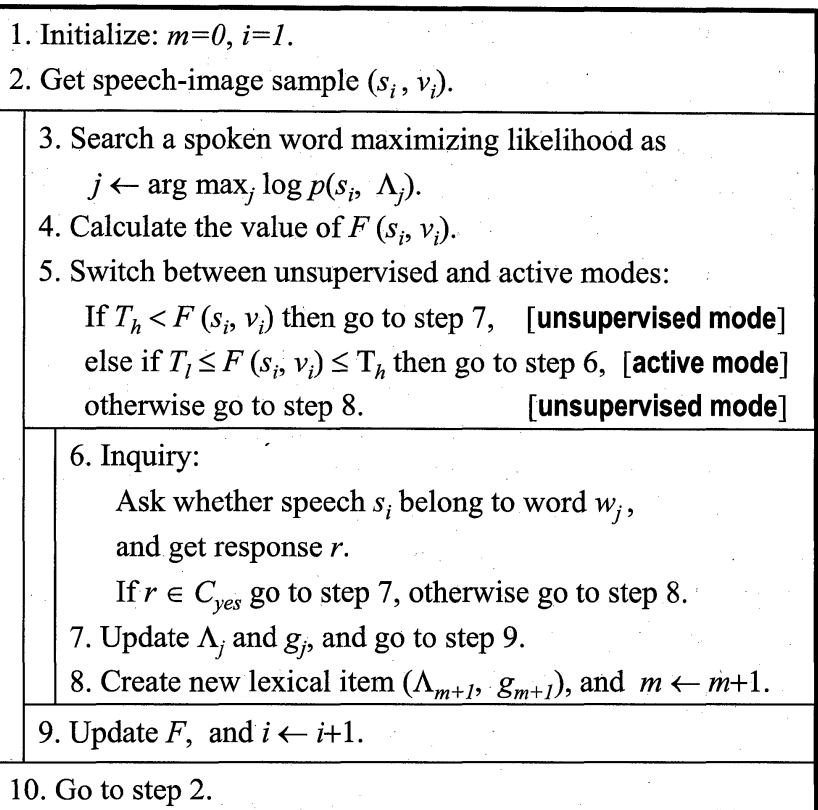

Fig. 6 Interactive learning algorithm.

$$
\frac{1}{1+\exp \left(-\alpha_{2}\left(H_{v}^{\prime}(v)-\beta_{2}\right)\right.}
$$

where

$$
\begin{aligned}
& H_{\nu}^{\prime}(v)=-\log p\left(v ; g_{j}\right), \\
& j=\arg \max _{k} \log p\left(s ; \Lambda_{u_{k}}\right)
\end{aligned}
$$

where $\alpha_{1}, \alpha_{2}, \beta_{1}$, and $\beta_{2}$ are the parameters of $F$, which are learned on-line. $H^{\prime}(v)$ is different from $H(v)$ in that the visual category is selected such that the corresponding word category whose likelihood is maximized with regard to the input speech $s$. In the experiment, if the value of $F$ was larger than the threshold $T_{h}$, the machine judged that the input speech $s$ was a known word $w_{j}$. If the value of $F$ was smaller than the threshold $T_{l}$, the machine judged that the input speech $s$ was an unknown word. If the value of $F$ fell between thresholds $T_{l}$ and $T_{h}$, an active learning mode was chosen, and the machine asked the user a question. In this mode, the machine output a speech sample for the word $w_{j}$, which had been input by the user and pre-recorded during the course of learning. This was taken to mean "Did you say the word $w_{j}$ ?" In response, the user answered yes or no. If the answer was yes, the machine took the input speech $s$ to be the known word $w_{j}$, and if the answer was no, the machine took $s$ to be an unknown word.

After making a decision about whether the input speech was a known or an unknown word, the machine updated its lexicon. If the input speech was judged to be an unknown word, the new word was added to the lexicon. In this word registration process, the model for the spoken words was set as a sequence of sub-word HMMs whose likelihood was maximized with regard to the speech used; the model for the image categories was set as a Gaussian calculated by maximum posterior probability estimation using the input im- 
age samples and a prior probability density of the Gaussian parameters. If the input speech was judged to be a known word, the existing statistical models for the word and its corresponding image category were updated. In this updating process, the HMM for the spoken word was replaced by a sequence of sub-word HMMs, the sum of whose likelihood values was maximized with regard to all speech samples that had been input into the machine and classified into this word. The Gaussian function for the image category was updated by using a given visual feature $v_{i}$ based on the maximum posterior probability learning.

The values of the parameters $\alpha_{1}, \alpha_{2}, \beta_{1}$, and $\beta_{2}$ in the confidence function $F$ were also learned on-line so that the output of $F$ approximated the probability that the input speech-image sample was one of the words in the lexicon. The values of the parameters were updated sample by sample based on the LMS algorithm according to the user's response $r$ as

$$
\begin{array}{r}
\alpha_{j} \leftarrow \alpha_{j}-\delta_{\alpha} \cdot \frac{\partial}{\partial \alpha_{i}}\left(1\left(r \in C_{y e s}\right)-F(s, v)\right)^{2}, \\
j=1,2, \\
\beta_{j} \leftarrow \beta_{j}-\delta_{\beta} \cdot \frac{\partial}{\partial \beta_{i}}\left(1\left(r \in C_{y e s}\right)-F(s, v)\right)^{2}, \\
j=1,2,
\end{array}
$$

where 1 is an indicator function

$$
1(B)= \begin{cases}1, & \text { if } B \text { is true } \\ 0, & \text { otherwise }\end{cases}
$$

and $C_{y e s}$ denotes a category of responses meaning yes. $\delta_{\alpha}$ and $\delta_{\beta}$ denote the learning rates.

\section{An Experiment with Interactive Spoken Language Acquisition}

Interactive spoken word acquisition was carried out by inputting speech-image pairs in the data set $X$ one by one in the order of the samples' index number. This order of samples means that the speech-image pairs for each of the forty words were input consecutively. This condition was set because a consecutive presentation of the same words would be natural and easily understandable to users when they try to teach new words to a machine. The values of the thresholds $T_{h}$ and $T_{l}$ were set to 0.8 and 0.1 , respectively. These values and the initial values of the parameters of the function $F$ were determined empirically so that the machine could frequently ask questions at the beginning of the learning course.

Figure 7 shows the changes in the sigmoid functions in the function $F$. Figure 8 shows the relative frequency of question asking for a ten-sample period, with questions divided into those answered with a yes and those answered with a no. From these figures, we can see that the relative frequency of question asking decreased as the function $F$ changed. The responses to the questions were all yes at the beginning. Then the proportions of yes and no

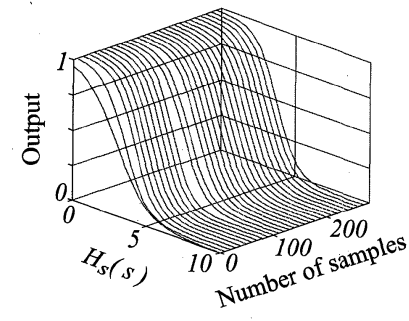

(a)

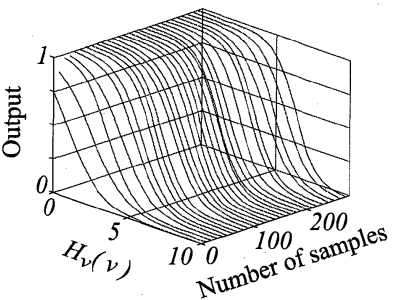

(b)
Fig. 7 Changes in function F. (a) Speech-input sigmoid function. (b) Image-input sigmoid function.

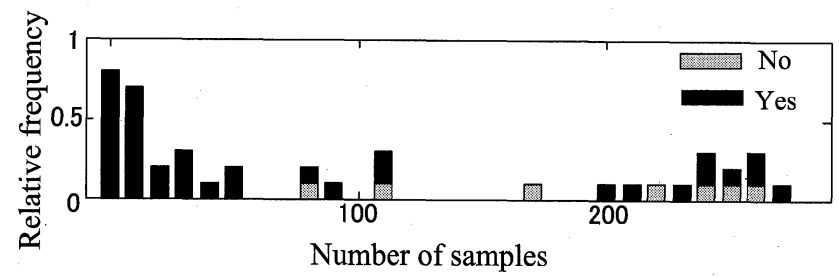

Fig. 8 Interaction during the course of learning.

answers gradually became equal. This means that each subsequent question provided more information. Eventually, for all speech-image pairs known/unknown word decisions were made correctly when each of them was input to the algorithm, and they were identified as the target lexical items corresponding to manually given labels.

In order to evaluate the validity of interaction, a wordacquisition experiment, in which a known/unknown decision was made without active learning, was done using the same learning data. In the experiment, input $(s, v)$ was decided to be a known word, when both speech-based and image-based decision measures, $H_{s}(s)$ and $H_{v}^{\prime}(v)$, were higher than the thresholds, 2.0 and 4.0, respectively, which were optimized empirically. From the results, forty-three lexical items were obtained, among which eight lexical items were learned incorrectly; the twenty-two samples for three word labels were classified as incorrect known words, and the samples for each of five word labels were separated into two different lexical items. This shows that it is not possible to achieve accuracy in learning without active learning.

In addition, in order to evaluate the effect of a consecutive presentation of the same words, an interactive wordacquisition experiment with a different order of input samples was carried out. In the experiment, as the opposite case of consecutive input, speech-image samples of each among forty words were input one-by-one in the order of $\left\{\left(s_{1}, v_{1}\right)\right.$, $\left.\left(s_{8}, v_{8}\right),\left(s_{15}, v_{15}\right), \ldots,\left(s_{273}, v_{273}\right),\left(s_{280}, v_{280}\right)\right\}$. In the result, forty lexical items were obtained, among which eight lexical items were learned incorrectly; eleven samples for six word labels were classified as incorrect known words, and the samples for one word label was separated into two different lexical items. The decision errors occurred when each of multiple image categories had been learned by only one sample, and had been not precise yet. We can see that cer- 
tain degree of consecutiveness of the same word in the input was necessary to achieve accuracy in the learning.

\section{Improving Learning Efficiency Through User Adap- tation}

So far, we have seen how the machine adapts to the input given by the user. In this section, we shall see how the method enables the user to adapt to the machine. An experiment was carried out in which the user's adaptive behaviour was simulated using a simple hypothetical rule. The hypothetical rule was this: If the machine has not asked questions for three consecutive presentations of the same word, then assume that the machine has learned the word sufficiently well and do not input any more speech-image samples for the word.

The speech-image samples in the data set $X$ were presented in the order of the samples' index number in the same way as in the experiment described in the previous section. Based on the above rule, when the machine did not ask questions for three consecutive presentations of the same word, the remaining samples for the same word were skipped. As a result, the learning of forty words was completed with the input of only 178 speech-image samples. The relative frequency of question asking is shown in Fig. 9.

In order to investigate whether image models were appropriately learned with a smaller number of training samples, a test experiment was carried out using the lexicon learned in the above learning experiment. In the experiment, the samples skipped in the course of learning were classified into lexical items such that the output of the confidence function was maximized as

$$
\begin{aligned}
c= & \arg \max _{1 \leq k \leq m}\left[\frac{1}{1+\exp \left(-\alpha_{1}\left(H_{s}(s ; k)-\beta_{1}\right)\right.}\right. \\
& \left.\frac{1}{1+\exp \left(-\alpha_{2}\left(H_{v}(v ; k)-\beta_{2}\right)\right.}\right]
\end{aligned}
$$

where

$$
\begin{aligned}
& H_{s}(s ; k)=\max _{q} \log p\left(s ; \Lambda_{q}\right)-\log p\left(s ; \Lambda_{u_{k}}\right) \\
& H_{v}(v ; k)=-\log p\left(v ; g_{k}\right),
\end{aligned}
$$

where $c$ and $m$ denote, respectively, the index of the lexical item to which input sample $(s, v)$ was classified and the number of lexical items in the lexicon. In this experiment, all the samples were correctly classified to their respective lexical items. This result shows the generalization performance of the method. In addition, distributions of the values of the image-based decision measure were calculated using the image models learned in the experiment with user adaptation, and these are shown in Fig. 10. We can see that the differences between the corresponding distributions in Figs. 4 (b) and 10 are small. We can thus conclude that appropriate image models were learned despite the use of a smaller number of learning samples. This also means that the machine's switching between active and unsupervised learning modes provides the user with information on the

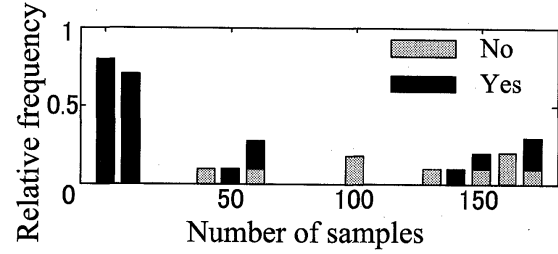

Fig. 9 Improvement obtained by simulating adaptive behaviour of the user.

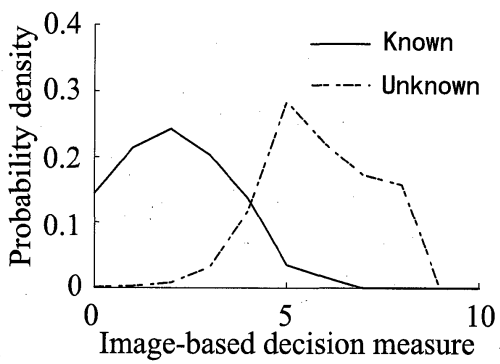

Fig. 10 Distribution of values of the image-based decision measure calculated using image models learned in the experiment with user adaptation.

machine's learning state. Users can thus avoid redundancy in learning by adapting to the machine's learning state.

\section{Discussion}

\subsection{Online Adaptation of Confidence Function}

In the interactive learning experiments, the proportions of yes and no answers to the questions asked by the user gradually became equal, which means that each subsequent question provided more information. This desirable outcome was a result of an adaptive change of the confidence function. Thus, the proposed method for online adaptation of the confidence function could effectively control the learning interaction.

\subsection{Speech and Visual Input}

The method used spoken words as speech input and static images of individual objects as visual input. In the future, it may be possible to increase the complexity of the input, e.g., from isolated words to continuous utterances. One way to achieve that is to replace the conventional speechbased unknown-word detection method used in continuous speech recognition with the proposed unknown word detection method.

The proposed method can also be embedded into a multimodal language learning method [19] which enables robots to understand the user's utterances and act appropriately in a given situation through multimodal interactive learning. This will make it possible to use multiple objects and dynamic images as visual input. 


\subsection{Visual Features}

The proposed method does not strongly depend on the selection of the visual features. In the proposed method, the simple visual features were used to make the validity of the method clear. How to improve the visual features should be investigated in the future in order to achieve higher scalability and generalization performance of the method. In terms of colour, the method uses the average colour of an object by assuming that such object colour is almost always monotint. More complex colour features, which represent multiple colours and textures of objects, could be used. In terms of shape, the method uses two-dimensional outline features by assuming that objects are shown in nearly the same directions. More complex shape features, which represent three-dimensional characteristics of objects, could be used.

\subsection{Predetermined Values}

Although many parameters used in the method are adaptive, there still remain some parameters whose values were predetermined manually. These include the initial values of the parameters $\alpha_{1}, \alpha_{2}, \beta_{1}$, and $\beta_{2}$ in the confidence function $F$; the thresholds $T_{h}$ and $T_{l}$ used by the machine to choose between the active and unsupervised modes; ' and the parameters used in the maximum posterior probability learning. Some of these values can be determined manually so that the machine can adapt to variations in the learning conditions, given the fact that the number of possible variations is limited due to physical constraints in the real world and finite human cognitive abilities. Other parameters may also become adaptive in the future.

\subsection{Response by Speech}

In the experiments, the user's yes/no response was input through a keyboard. When the keyboard input is replaced with a speech interface, the misrecognition of speech has an influence on word-acquisition performance. This influence should be investigated in the future.

\subsection{Scalability}

The learning becomes more difficult as the number of lexical items to be learned increases. The problem is that both the acoustic and visual distances between lexical items are small on average in such cases. However, it should be pointed out that even if the acoustic differences between lexical items are very ambiguous, the proposed method would be able to compensate for such ambiguity by using the visual differences, and thus improve performance.

\subsection{Effect on the User's Behaviour}

The results presented in the previous sections suggest that a combination of active and unsupervised learning modes provides a natural way for a user and a machine to adapt to each other. The switching between active and unsupervised modes appears to affect not only the way of selecting words as described in the previous section but also other forms of user behaviour in interactive interfaces including the way of speaking and the way of presenting objects. The effect of applying the combination of active and unsupervised learning modes on the user behaviour should be investigated further.

\section{Conclusion}

This paper described a new word acquisition method that enhances multimodal interfaces. The method is based on a combination of active and unsupervised learning principles. This combination has been found to provide for natural and efficient human-machine interaction, which in turn enables the user and the machine to adapt to each other in a word acquisition task. This combination of active and unsupervised learning will play an important role in mutual human-machine adaptation in other general interactive learning tasks and should be investigated further.

\section{Acknowledgements}

I would like to thank Kenji Nagatomo, Takayuki Nagai, and Akira Kurematsu for their help and support. This research was supported in part by the National Institute of Information and Communications Technology.

\section{References}

[1] D. Sperber and D. Wilson, Relevance, 2nd ed., Blackwell, 1995.

[2] P. Bloom, How children learn the meanings of words, MIT Press, 2000.

[3] A. Gorin, S. Levinson, and A. Sanker, "An experiment in spoken language acquisition," IEEE Trans. Speech Audio Processing, vol.2, no.1, pp.224-240, 1994.

[4] S. Nakagawa, H. Nakanishi, Y. Kobu, and M. Itahashi, "Concept acquisition based on combining with visual and auditory information," Journal of Artificial Intelligence, vol.8, no.4, pp.109-118, 1993.

[5] S. Akaho, S. Hayamizu, O. Hasegawa, T. Yoshimura, and H. Asoh, "Concept acquisition from multiple information sources by the EM algorithm," IEICE Trans. Fundamentals (Japanese Edition), vol.J80A, no.9, pp.1546-1553, Sept. 1997.

[6] D. Roy, Learning words from sights and sounds: A computational model, Ph.D. Thesis, MIT, 1999.

[7] A. Gorin, D. Petrovska-Delacretaz, G. Riccardi, and J. Wright, "Learning spoken language without transcriptions," Proc. IEEE Workshop Speech Recognition and Understanding, vol.1, pp.293296, 1999.

[8] N. Iwahashi, "Language acquisition through a human-robot interface by combining speech, visual, and behavioral information," Inf. Sci., vol.156, pp.109-121, 2003.

[9] T. Inamura, N. Inaba, and H. Inoue, "Integration model of learning mechanism and dialogue strategy based on stochastic experience representation using bayesian network," Proc. Int. Workshop on Robot and Human Interactive Communication, pp.247-252, 2000.

[10] L. Steels and K. Kaplan, "AIBO's first words: The social learning of language and meaning," Evolution of Communication, vol.4, no.1, pp.3-32, 2001.

[11] R. Taguchi, M. Kimura, S. Shinohara, Y. Iribe, K. Katsurada, and T. 
Nitta, "Efficient learning of word meanings by agents using biases observed in language development of children," Journal of Artificial Intelligence, vol.22, no.3, pp.444 453, 2007.

[12] S. Dusan and J. Flanagan, "Adaptive dialog based multimodal language acquisition," Proc. International Conference on Multimodal Interfaces, 2002.

[13] D. Ballard and C. Yu, "A multimodal learning interface for word acquisition," Proc. Int. Conf. on Acoustics, Speech and Signal Processing, vol.5, pp.784-787, 2003.

[14] N. Iwahashi, "Active and unsupervised learning of spoken words through a multimodal interface," Proc. IEEE Workshop on Robot and Human Interactive Communication, pp.437-442, 2004.

[15] S. Davis and P. Mermelstein, "Comparison of parametric representations for monosyllabic word recognition in continuously spoken sentences," IEEE Trans. Acoust. Speech Signal Processing, vol.28, no.4, pp.357-366, 1980.

[16] E. Persoon and K.S. Fu, "Shape discrimination using Fourier descriptors," IEEE Trans. Syst. Man Cybern., vol.7, no.3, pp.170-179, 1977.

[17] S. Nakagawa, M. Saito, and M. Masukata, "Automatic acquisition of speech processing units and its application to speech recognition/speech coding," Journal of Artificial Intelligence, vol.13, no.4, pp.115-126, 1998.

[18] L.E. Baum and T. Petrie, "Statistical inference for probabilistic function of finitc state markov chain," Annals of Mathematical Statistics, vol.37, no.6, pp.1554-1563, 1966.

[19] N. Iwahashi, "A method of coupling of belief systems through human-robot language interaction," Proc. IEEE Workshop on Robot and Human Interactive Communication, pp.385-390, 2003.

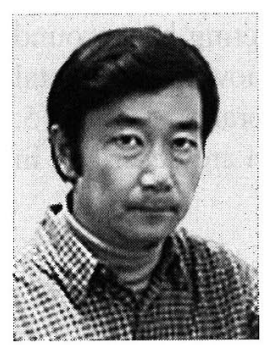

Naoto Iwahashi received the B.E. degree in engineering from Keio University in 1985, Yokohama, Japan. He received Ph.D. degree in engineering from Tokyo Institute of Technology in 2001. In 1985, he joined Sony Corporation, Tokyo, Japan. From 1990 to 1993, he stayed at ATR Interpreting Telephony Research Laboratories, Kyoto, Japan. From 1998 to 2003, he was with Sony Computer Science Laboratories Inc., Tokyo, Japan. In 2003, he joined Advanced Telecommunications Research Laboratories International. In 2006, he joined National Institute of Information and Communications Technology. His research areas include interactive speech system, language acquisition, human-robot interaction. 